\title{
VIRIDANS GROUP STREPTOCOCCI IN LEUKAEMIA AND STEM CELL TRANSPLANT: REVIEW OF A RISK-STRATIFIED GUIDELINE FOR EMPIRIC VANCOMYCIN IN FEBRILE NEUTROPAENIA
}

\author{
Sandra Ruhayel ${ }^{1}$, David Foley ${ }^{2}$, Kate Hamilton ${ }^{3}$, Patricia Ferguson ${ }^{3}$, Rishi Kotecha ${ }^{2}$, Asha \\ Bowen $^{4}$, and Daniel Yeoh ${ }^{2}$ \\ ${ }^{1}$ Perth Children's Hospital Department of Paediatric and Adolescent Oncology and \\ Haematology \\ ${ }^{2}$ Perth Children's Hospital \\ ${ }^{3}$ Westmead Hospital \\ ${ }^{4}$ THe university of Western Australia, WA Centre for Child Health Research
}

November 16, 2020

\begin{abstract}
Viridans group streptococci (VGS) are an important cause of sepsis in children with cancer. Penicillin-resistance amongst VGS isolates is increasing. At our institution, vancomycin is added to standard empiric therapy for febrile neutropaenia in patients at high-risk of VGS sepsis. We reviewed effectiveness of this risk-stratified approach by examining episodes of VGS bacteraemia over three years. In 19 VGS bloodstream infection episodes, $100 \%$ of isolates from high-risk patients and $78.9 \%$ of all episodes were susceptible to risk-stratified empiric antibiotics. Based on blood culture time-to-positivity, empiric vancomycin can be safely discontinued in stable patients with no growth at 24 hours.
\end{abstract}

\section{INTRODUCTION}

Viridans group streptococci (VGS) are an important cause of bacteraemia in children with cancer. ${ }^{1-4}$ VGS bloodstream infection (VGS-BSI) can rapidly progress to cause a toxic shock-like syndrome characterised by hypotension and acute respiratory distress syndrome, ${ }^{5-7}$ with mortality of up to $23 \%$ in children. ${ }^{5,7,8}$ Groups at highest risk of VGS-BSI include children with acute myeloid leukaemia (AML), ${ }^{4}$ relapsed acute lymphoblastic leukaemia (ALL), ${ }^{9}$ infant ALL, ${ }^{10}$ and those undergoing haematopoietic stem cell transplant (HSCT). ${ }^{11}$

VGS-BSI associated mortality, coupled with a rise in penicillin-resistance amongst VGS isolates, ${ }^{12}$ has led to advocacy for the addition of empiric vancomycin to treat febrile neutropaenia in high-risk children. ${ }^{13}$ However, recommendations differ between guidelines and practice varies between centres. ${ }^{14,15}$ At our institution, the addition of empiric vancomycin for the initial management of febrile neutropaenia is recommended in patients at highest-risk of VGS-BSI. We aimed to assess the safety and efficacy of this risk-stratified approach by examining the epidemiology, microbiology, and outcomes of VGS-BSI at our centre.

\section{METHODS}

We conducted a single-centre prospective cohort study including all children (0-18 years) receiving chemotherapy for haematological malignancy or undergoing HSCT from $1^{\text {st }}$ November 2016 until $31^{\text {st }}$ January 2020 
at Perth Children's Hospital in Western Australia. As per institutional guidelines, addition of empiric vancomycin to standard febrile neutropaenia therapy (piperacillin-tazobactam) is recommended in all patients at highest-risk of VGS-BSI (those with AML, relapsed ALL, infant ALL or undergoing HSCT), and any patient with clinical signs of shock. Shock was defined as inadequate cardiac output with hypotension requiring intravascular volume expansion (fluid bolus [?] $20 \mathrm{ml} / \mathrm{kg}$ ) and/or inotropic support. Neutropaenia was defined as an absolute neutrophil count of $<0.5 \times 10^{9} / \mathrm{L}$. Fever was defined as core body temperature [?] $38.5^{\circ} \mathrm{C}$ on a single occasion or [?]38.0degC on two sequential occasions within a 24-hour period.

All VGS-BSI episodes were identified from the laboratory information database of blood culture isolates. A VGS-BSI episode was defined as isolation of Viridans group Streptococcus spp. from a blood culture. Episodes with concomitant growth of a gram-positive commensal (e.g. Micrococcus spp. or coagulase negativeStaphylococcus ) were excluded as contaminants.

For each VGS-BSI episode, demographic and clinical data were collected from patient medical records including the presence of shock, requirement for mechanical ventilation, admission to the intensive care unit (ICU), and survival at day 7 and day 30. Laboratory data included blood culture time-to-positivity and antibiotic susceptibility. Susceptibility testing was carried out using an E-test? (Biomerieux, France) as per manufacturer specifications. ${ }^{16}$ Susceptibility to empiric B-lactam antibiotic (piperacillin-tazobactam) was inferred from penicillin minimum inhibitory concentration, with breakpoints defined as per the Clinical \& Laboratory Standards Institute. ${ }^{17}$

Statistical analysis was performed using GraphPad QuickCalcs (GraphPad Software, Inc., CA, US). Fisher's exact test was used to compare the characteristics of high-risk and non-high-risk patients for categorical data, and the Mann-Whitney $U$ test for continuous variables. $\mathrm{P}$ values of $<0.05$ were considered statistically significant. National ethics approval was granted by the Western Sydney Local Health District Human Research Ethics Committee (HREC/16/WMEAD/281).

\section{RESULTS}

Of 84 total BSI episodes during the study period, 19 (22.6\%) were due to VGS (Table 1). Amongst patients with VGS-BSI, median age was 6.7 (interquartile range (IQR) 2.3-10.7) years and $63.2 \%(12 / 19)$ of episodes occurred in females. The majority $(57.9 \%, 11 / 19)$ of VGS-BSI episodes occurred in high-risk patients; 7 with AML, 3 post-HSCT, and in one child with ALL and clinical evidence of shock.

Of the VGS isolates, $21.1 \%$ (4/19) were penicillin-resistant, and $36.8 \%(7 / 19)$ had reduced penicillin susceptibility. The median blood culture time-to-positivity was 11.7 (IQR $9.1-13.8$ ) hours; $100 \%$ flagged positive within 17 hours (Fig. 1). VGS isolates were susceptible to recommended risk-stratified empiric antibiotics in $100 \%$ (11/11) of isolates from high-risk patients and $78.9 \%(15 / 19)$ of all episodes. In all non-high-risk, clinically stable patients with a penicillin-resistant VGS isolate (4/19), vancomycin was commenced once the blood culture flagged positive, within 24 hours of fever onset.

All VGS-BSI episodes were associated with fever, and $89.5 \%(17 / 19)$ of patients had neutropaenia. One patient developed intracranial haemorrhage secondary to a leukaemia-related coagulopathy and died in ICU shortly after, unrelated to the VGS-BSI. All other patients recovered without requiring ICU admission; there were no VGS-BSI related deaths. No patients with VGS-BSI had vancomycin associated nephrotoxicity.

\section{DISCUSSION}

VGS-BSI can cause life-threatening complications in children with cancer and early initiation of appropriate antibiotic therapy is essential. With increasing penicillin-resistance amongst VGS isolates, ${ }^{12}$ febrile neutropaenia monotherapy with an extended spectrum B-lactam agent may be suboptimal in patients at high risk of VGS-BSI. ${ }^{13}$ However, the universal addition of vancomycin to empiric febrile neutropaenia therapy has not shown benefit, increases nephrotoxicity, ${ }^{18}$ and the emergence of resistance, ${ }^{19}$ and is not routinely recommended. ${ }^{15,20} \mathrm{~A}$ risk-stratified approach limits the addition of empiric vancomycin to febrile neutropaenia therapy for high-risk and clinically unstable patients, maximising utility and minimising the potential 
for harm. This report demonstrates that this approach is safe and effective, guiding appropriate therapy and supporting good clinical outcomes in VGS-BSI.

The proportion of non-penicillin susceptible VGS isolates (57.9\%) was comparable to previous paediatric studies (29.6\% to $67.5 \%) \cdot{ }^{6,9,21}$ Reassuringly, there were no VGS-BSI related deaths and all VGS isolates in high-risk patients were susceptible to the recommended empiric antibiotics. Whilst four non-high-risk patients had a non-penicillin susceptible VGS-BSI, vancomycin was added within 24 hours and all recovered without incident. The clinical stability observed in these patients, despite the slight delay in vancomycin therapy, may attest to the lower pathogenicity of VGS in lower risk populations.

Modern laboratory methods allow continuous assessment of blood culture samples, allowing early notification and time-to-positivity assessment. In this study, all blood cultures were detected as positive for VGS-BSI within 17 hours, indicating that empiric vancomycin targeting VGS can be safely discontinued in stable patients at 24 hours if cultures remain negative.

This study is limited by the single-centre design and small sample size but given the limited data assessing such a risk-stratified approach, the findings remain important. Secondly, we employed a pragmatic definition of BSI including all single VGS isolates, which may have led to an overestimation of VGS-BSI episodes. The definition of VGS-BSI is not standardised and whilst some previous surveillance studies required isolation of VGS from two samples, ${ }^{7,9}$ several other studies have defined VGS-BSI as any single isolation of VGS VG, $^{6,22}$ as

this would result in treatment initiation from a clinical perspective. Notably, all VGS-BSI episodes in this study were associated with fever and were treated as true episodes of bacteremia. ${ }^{22}$

In conclusion, this data demonstrates the safety of a risk-stratified approach targeting the addition of empiric vancomycin to febrile neutropaenia therapy in children with cancer at high-risk of VGS-BSI. The majority of patients received appropriate antibiotic cover for VGS-BSI and no episodes progressed to VGS-BSI related shock requiring ICU admission; there were no VGS-BSI associated deaths. All VGS isolates flagged positive within 24 hours indicating empiric vancomycin can be discontinued after 24 hours in clinically stable patients. Broader assessment of this risk-stratified approach is warranted.

\section{CONFLICT OF INTEREST:}

The authors declare that there is no conflict of interest to disclose.

\section{ACKNOWLEDGEMENTS:}

We would like to acknowledge the Children's Antimicrobial Stewardship Program at Perth Children's Hospital, as well as the Nurse Consultants of the Infection Prevention and Control team (in particular Dallas Sewell and Stacey Fitzgerald). We would like to thank the larger team behind the "Bacteraemia in Malignant Haematology and Haematopoietic Stem Cell Transplantation: A Multicentre Registry" through the ASID Clinical Research Network.

\section{REFERENCES}

1. Reilly AF, Lange BJ. Infections with viridans group streptococci in children with cancer. Pediatr Blood Cancer. 2007;49(6):774-780.

2. Lewis V, Yanofsky R, Mitchell D, et al. Predictors and outcomes of viridans group streptococcal infections in pediatric acute myeloid leukemia: from the Canadian infections in AML research group.Pediatr Infect Dis J. 2014;33(2):126-129.

3. Mikulska M, Viscoli C, Orasch C, et al. Aetiology and resistance in bacteraemias among adult and paediatric haematology and cancer patients. The Journal of infection. 2014;68(4):321-331.

4. Persson L, Vikerfors T, Sjoberg L, Engervall P, Tidefelt U. Increased incidence of bacteraemia due to viridans streptococci in an unselected population of patients with acute myeloid leukaemia. Scand J Infect Dis. 2000;32(6):615-621. 
5. Huang WT, Chang LY, Hsueh PR, et al. Clinical features and complications of viridans streptococci bloodstream infection in pediatric hemato-oncology patients. J Microbiol Immunol Infect.2007;40(4):349354 .

6. Ahmed R, Hassall T, Morland B, Gray J. Viridans Streptococcus bacteremia in children on chemotherapy for cancer: an under-estimated problem. Pediatr Haematol Oncol. 2003(20):439-444.

7. Gassas A, Grant R, Richardson S, et al. Predictors of viridans streptococcal shock syndrome in bacteremic children with cancer and stem-cell transplant recipients. J Clin Oncol.2004;22(7):1222-1227.

8. Brunet AS, Ploton C, Galambrun C, et al. Low incidence of sepsis due to viridans streptococci in a ten-year retrospective study of pediatric acute myeloid leukemia. Pediatr Blood Cancer. 2006;47(6):765-772.

9. Nielsen MJ, Claxton S, Pizer B, et al. Viridans Group Streptococcal Infections in Children After Chemotherapy or Stem Cell Transplantation: A 10-year Review From a Tertiary Pediatric Hospital. Medicine (Baltimore). 2016;95(9):e2952.

10. Salzer W, Dinndorf P, Dreyer Z, Hilden J, Reaman GH. Analysis of infectious complications in infants with acute lymphoblastic leukemia treated on the Children's Cancer Group Protocol 1953: a report from the Children's Oncology Group. J Pediatr Hematol Oncol.2009;31(6):398-405.

11. Martino R, Manteiga R, Sanchez I, et al. Viridans streptococcal shock syndrome during bone marrow transplantation. Acta Haematol.1995;94(2):69-73.

12. Bruckner L, Gigliotti F. Viridans group streptococcal infections among children with cancer and the importance of emerging antibiotic resistance. Semin Pediatr Infect Dis. 2006;17(3):153-160.

13. Adcock KG, Akins RL, Farrington EA. Evaluation of empiric vancomycin therapy in children with fever and neutropenia. Pharmacotherapy.1999;19(11):1315-1320.

14. Freifeld AG, Bow EJ, Sepkowitz KA, et al. Clinical Practice Guideline for the Use of Antimicrobial Agents in Neutropenic Patients with Cancer: 2010 Update by the Infectious Diseases Society of America. Clinical Infectious Diseases. 2011;52(4):e56-e93.

15. Lehrnbecher T, Robinson P, Fisher B, et al. Guideline for the Management of Fever and Neutropenia in Children With Cancer and Hematopoietic Stem-Cell Transplantation Recipients: 2017 Update. J Clin Oncol. 2017;35(18):2082-2094.

16. Biomeriux. E-test product information. https://www.biomerieux.com.au/products/etestr. Published 2018. Updated May 2018. Accessed 31/05/2020, 2020.

17. Clinical and Laboratory Standards Institute. M100: Performance Standards for Antimicrobial Susceptibility Testing. Published 2020. Accessed 01/09/2020, 2020.

18. Beyar-Katz O, Dickstein Y, Borok S, Vidal L, Leibovici L, Paul M. Empirical antibiotics targeting gram-positive bacteria for the treatment of febrile neutropenic patients with cancer. The Cochrane database of systematic reviews. 2017;6(6):CD003914.

19. Karandikar MV, Milliren CE, Zaboulian R, et al. Limiting Vancomycin Exposure in Pediatric Oncology Patients With Febrile Neutropenia May Be Associated With Decreased Vancomycin-Resistant Enterococcus Incidence.J Pediatric Infect Dis Soc. 2020;9(4):428-436.

20. Freifeld AG, Bow EJ, Sepkowitz KA, et al. Clinical practice guideline for the use of antimicrobial agents in neutropenic patients with cancer: 2010 Update by the Infectious Diseases Society of America.Clinical infectious diseases : an official publication of the Infectious Diseases Society of America. 2011;52(4):427431.

21. Kennedy HF, Gemmell CG, Bagg J, Gibson BE, Michie JR. Antimicrobial susceptibility of blood culture isolates of viridans streptococci: relationship to a change in empirical antibiotic therapy in febrile 
neutropenia. J Antimicrob Chemother. 2001;47(5):693-696.

22. Dulanto Chiang A, Sinaii N, Palmore TN. Risk Factors for Viridans Group Streptococcal Bacteremia in Neutropenic and Non-neutropenic Patients: A Single Center Case-Case-Control Study. Open Forum Infect Dis. 2018;5(1):ofx260.

\section{LEGENDS}

TABLE 1 Comparison of diagnosis, demographics, clinical data and treatment, microbiological data between high-risk and non-high-risk patients with VGS-BSI ( $\mathrm{n}=19)$. Abbreviations: AML, acute myeloid leukaemia; HSCT, haematopoietic stem cell transplant; ALL, acute lymphoblastic leukaemia; IQR, interquartile range; BC, blood culture; ICU, intensive care unit; VGS-BSI, viridans group streptococci-bloodstream infection.

FIGURE 1 Graph of percentage (\%) of total VGS blood cultures (BC) flagging positive versus time-topositivity (TTP) in hours. $89 \%$ flagged positive within 14 hours, $95 \%$ within 15 hours and $100 \%$ within 17 hours.

\section{Hosted file}

Table1_Results_16112020.pdf available at https://authorea.com/users/355150/articles/493236viridans-group-streptococci-in-leukaemia-and-stem-cell-transplant-review-of-a-riskstratified-guideline-for-empiric-vancomycin-in-febrile-neutropaenia 


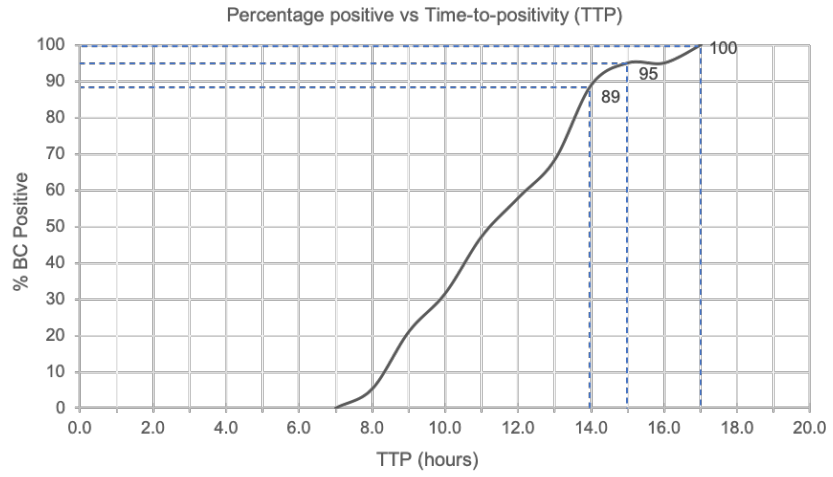

